\title{
Relación entre el consumo de grasas vegetales insaturadas y el contenido de ácidos grasos de la leche de ovejas
}

\author{
Martínez Marín, A.L. ${ }^{\circledR} ;$ Núñez Sánchez, N. ${ }^{\text {; }}$ Pavón Vijande, R. ${ }^{\text {; }}$ Gómez Díaz, R. ${ }^{2}$; Peña Blanco, F. ${ }^{1}$ y García Martínez, A. ${ }^{1}$ \\ 'Departamento de Producción Animal. Universidad de Córdoba. España. \\ 2Departamento de Bromatología y Tecnología de Alimentos. Universidad de Córdoba. España.
}

\section{PALABRAS CLAVE ADICIONALES}

Aceites.

Semillas.

Grasa láctea.

Metarregresión.

\section{RESUMEN}

El efecto de la inclusión de aceites y semillas, ricos en ácidos grasos insaturados, en la dieta de ovejas sobre el contenido de ácidos grasos de la grasa láctea se cuantificó mediante metarregresión. Se utilizaron los resultados de 22 trabajos de investigación con un total de 67 tratamientos. Los trabajos se realizaron con 10 razas ovinas europeas y americanas que recibieron dietas con hasta un $7 \%$ de grasa insaturada. Los contenidos de los ácidos grasos saturados de cadena media (AGSCM) mostraron una respuesta lineal negativa al consumo creciente de grasa, que fue más acentuada en el caso del ácido palmítico. Los contenidos del ácido esteárico y de los ácidos grasos insaturados de 18 átomos de carbono (AGI-C18) aumentaron de forma lineal, pero la respuesta fue mayor en los segundos. Los cambios en los AGSCM y AGI-C18 ocasionaron que el índice de aterogenicidad disminuyera linealmente en la grasa láctea. Estos resultados indican que la inclusión de fuentes de grasa ricas en ácidos insaturados en dietas para ovejas ocasiona un cambio favorable en la composición de ácidos grasos de la grasa láctea desde el punto de vista de la salud humana.

\section{Relationship between unsaturated lipid intake and fatty acid contents in sheep milk fat}

\section{SUMMARY}

A meta-regression study was conducted to quantify the effects of adding unsaturated plant lipids to ewe's diet on the fatty acid contents in milk fat. The results used in the study were obtained from 22 investigations, comprising 67 treatments, carried out with 10 American and European sheep breeds. Extra fat in the experimental treatments was up to $7 \%$. Medium chain saturated fatty acids (MCSFA) contents showed a negative linear response to the increasing fat intake, which was more accentuated in the case of palmitic acid. The contents of stearic acid and, even more, the sum on unsaturated fatty acids with 18 atoms of carbon (C18-UFA) linearly increased with fat intake. Due to the observed changes in MCSFA and C18-UFA, the atherogenicity index of milk fat linearly decreased. These results indicate that the inclusion of plant oils and oilseeds rich in unsaturated fatty acids in the diet of ewes has a positive effect on the milk fatty profile from the human's health point of view.

\section{INFORMACIÓN}

Cronología del artículo.

Recibido/received: 16.04 .15

Aceptado/Accept: 21.02.16

Online: 15.09 .16

Correspondencia a los autores/Contact e-mail:

palmartm@uco.es

\section{INTRODUCCIÓN}

Algunos autores han estudiado el efecto de la adición de diferentes cantidades de grasas insaturadas de origen vegetal a la dieta de ovejas sobre el perfil de ácidos grasos de la grasa láctea (Zhang et al., 2006c; GómezCortés et al., 2009; Gómez-Cortés et al., 2011b). Sin embargo, ninguno de ellos ha investigado la relación entre la magnitud de la respuesta observada y la cantidad de grasa extra consumida. Por otra parte, en la bibliografía, las cantidades de lípidos insaturados suministrados a las ovejas cubren un amplio rango, por ejemplo de 60 a 165 g/d (Gómez-Cortés et al., 2011b). Por tanto, un meta-análisis de los trabajos de investigación disponibles sería una alternativa a la falta de información sobre el tema. El meta-análisis es una herramienta estadística usada para revisar de manera objetiva los resultados de investigaciones previas sobre un tema, 
Tabla I. Trabajos de investigación incluidos en la metarregresión para el estudio de la relación entre el consumo de grasas vegetales insaturadas y el contenido de ácidos grasos de la leche de ovejas (Experimental works whose results were included in the meta-regression study to quantify the effects of adding unsaturated plant lipids to ewe's diet on the fatty acid contents in milk fat).

\begin{tabular}{|c|c|c|c|c|}
\hline Trabajo & Año & Autor & Experimentos & $\begin{array}{l}\text { Tratamientos } \\
\text { por experimento }\end{array}$ \\
\hline 1 & 2004 & Chiofalo, B. et al. Small Rum Res, 55: 169-176 & 1 & 2 \\
\hline 2 & 2006 & Mele, M. et al. Anim Res, 55: 273-285 & 1 & 4 \\
\hline 3 & $2006 a$ & Zhang, R.H. et al. Small Rum Res, 63: 233-241 & 1 & 4 \\
\hline 4 & $2006 b$ & Zhang, R.H. et al. Anim Feed Sci Technol, 127: 220-233 & 1 & 3 \\
\hline 5 & $2006 c$ & Zhang, R.H. et al. Can J Anim Sci, 86: 547-556 & 1 & 4 \\
\hline 6 & 2007 & Bouattour, M. Tesis. UAB. Barcelona. & 1 & 3 \\
\hline 7 & $2008 a$ & Gómez-Cortés, P. et al. J Dairy Sci, 91: 1560-1569 & 1 & 2 \\
\hline 8 & $2008 b$ & Gómez-Cortés, P. et al. J Dairy Sci, 91: 3119-3127 & 1 & 2 \\
\hline 9 & 2008 & Hervás, G. et al. J Dairy Res, 75: 399-405 & 1 & 2 \\
\hline 10 & 2009 & Castro, T. et al. Small Rum Res, 84: 47-53 & 1 & 2 \\
\hline 11 & 2009 & Gómez-Cortés, P. et al. J Dairy Sci, 92: 4122-4134 & 1 & 3 \\
\hline 12 & 2010 & Cieslak, A. et al. Czech J Anim Sci, 55: 496-504 & 2 & 3 \\
\hline 13 & $2010 b$ & Toral, P.G. et al. J Dairy Sci, 93: 1655-1667 & 1 & 2 \\
\hline 14 & $2010 a$ & Toral, P.G. et al. J Dairy Sci, 93: 1604-1615 & 1 & 2 \\
\hline 15 & 2011 & Caroprese, M. et al. J Dairy Sci, 94: 3856-3867 & 1 & 4 \\
\hline 16 & 2011a & Gómez-Cortés, P. et al. J Dairy Sci, 94: 4578-4588 & 1 & 6 \\
\hline 17 & $2011 b$ & Gómez-Cortés, P. et al. Food Chem, 125: 644-651 & 1 & 4 \\
\hline 18 & 2011 & De Oliveira Maia, M. Tesis. Sao Paulo. Piracicaba. & 1 & 3 \\
\hline 19 & 2011 & Maia Ferreira, E. Tesis. Sao Paulo. Piracicaba. & 1 & 2 \\
\hline 20 & 2012 & Paduan Nolli, C. Tesis. Sao Paulo. Piracicaba. & 1 & 3 \\
\hline 21 & 2012 & Mughetti, L. et al. Anim Feed Sci Technol, 178: 27-39 & 1 & 3 \\
\hline
\end{tabular}

con independencia de la metodología empleada por los distintos autores. La metarregresión es un tipo de metaanálisis que permite integrar resultados cuantitativos para crear modelos que expliquen las observaciones (Sauvant et al., 2008).

El uso del meta-análisis ha permitido extraer valiosas conclusiones cuando se ha aplicado a experimentos de nutrición animal (Eugène et al., 2008; Sales y Homolka, 2011).

El objetivo de este trabajo fue cuantificar la relación entre la cantidad de grasa suplementaria consumida y los contenidos de los ácidos grasos de la leche ovina a partir de trabajos de investigación sobre la adición de lípidos vegetales insaturados a la dieta de ovejas.

\section{MATERIAL Y MÉTODOS}

Los trabajos de investigación se buscaron en bases de datos, utilizando como palabras clave grasas vegetales, semillas, aceites, ovejas, leche, grasa y ácidos grasos en los idiomas español, inglés, francés y portugués. Se seleccionaron solamente aquellos trabajos en los que un tratamiento control con una dieta sin grasa añadida se comparó con uno o más tratamientos en los que la dieta fue enriquecida con una fuente de grasa rica en ácidos grasos

Tabla II. Contenido en ácidos grasos mayoritarios (media \pm desviación típica), expresado en porcentaje de los ácidos grasos totales, de las fuentes de grasa utilizadas en los experimentos incluidos en la metarregresión para el estudio de la relación entre el consumo de grasas vegetales insaturadas y el contenido de ácidos grasos de la leche de ovejas (Major fatty acid contents (mean $\pm S D$ ), expressed as percentage of total fatty acids, in the plant oils and oilseeds that used in the investigations included in the meta-regression study to quantify the effects of adding unsaturated plant lipids to ewe's diet on the fatty acid contents in milk fat).

\begin{tabular}{lccr}
\hline Ácidos grasos & \multicolumn{2}{c}{ GO } & Fuentes de grasa \\
& & $G L$ & GAL \\
\hline Ácido oleico (C18:1 cis-9) & $66,1 \pm 13,3$ & $26,0 \pm 8,2$ & $20,3 \pm 0,8$ \\
Ácido linoleico (C18:2 cis-9,cis-12) & $15,5 \pm 6,3$ & $55,0 \pm 5,1$ & $15,3 \pm 2,2$ \\
Ácido $\alpha$-linolénico (C18:3 n-3) & $6,2 \pm 3,1$ & $2,1 \pm 3,0$ & $52,5 \pm 1,5$ \\
\hline
\end{tabular}

GO, GL y GAL= fuentes de grasa ricas en ácido oleico, ácido linoleico y ácido $\alpha$-linolénico, respectivamente. 
insaturados (AGI) y no protegida frente a la digestión ruminal. Se encontraron 22 trabajos de investigación, realizados entre los años 2004 y 2012, que incluyeron 23 experimentos y un total de 67 tratamientos (tabla I). En dichos trabajos se utilizaron 10 razas ovinas europeas y americanas que recibieron dietas con valores de grasa añadida, en forma de aceites y semillas oleaginosas, comprendidos entre un 1 y $7 \%$ (3,2 $\pm 1,5 \%)$. Los tratamientos se codificaron como NOLIP cuando la dieta no contenía grasa añadida (controles; $n=27)$ y como LIP $(n=40)$ si la dieta contenía grasa extra, proveniente de aceites ( $\mathrm{n}=$ $25)$ o semillas $(n=15)$ ricos en ácido oleico $(n=7)$, linoleico $(\mathrm{n}=19)$, o $\alpha$-linolénico $(\mathrm{n}=14)$ (tabla II). Las variables estudiadas se seleccionaron por su repercusión en la salud humana e incluyeron los contenidos de los ácidos grasos saturados de cadena corta y media (C6:0 a C16:0), el ácido esteárico, la suma de los ácidos oleico, linoleico y $\alpha$-linolénico (AGI-C18) y el índice de aterogenicidad (IA).

Los análisis estadísticos se hicieron con el programa SAS 9.1 (SAS Inst Inc, Cary, NC). Se utilizó el procedimiento MIXED de acuerdo con los modelos propuestos por Sauvant et al. (2008). Para la metarregresión, se comprobó mediante análisis gráfico de los datos (dispersión de los valores en una gráfica de coordenadas) que las únicas relaciones entre las variables dependientes estudiadas y el consumo de grasa eran lineales, por lo que solamente este tipo de relación fue investigada. El trabajo experimental se incluyó en la declaración CLASS del modelo y no contuvo ninguna información cuantitativa. Los datos se ponderaron utilizando la raíz cuadrada del número de animales usados en cada tratamiento mediante la declaración WEIGHT. El residual estudentizado y la distancia de Cook de cada observación se utilizaron para identificar los valores anómalos. Para cada ecuación obtenida se calculó la raíz cuadrada del error cuadrático medio (RECM) y el coeficiente de determinación $\left(\mathrm{R}^{2}\right)$.

\section{RESULTADOS Y DISCUSIÓN}

El peso vivo medio de las ovejas utilizadas en los trabajos experimentales fue $66,4 \pm 15,6 \mathrm{~kg}$. El consumo medio de grasa en el tratamiento LIP fue de 81,6 $6 \pm 32,4$ $\mathrm{g} / \mathrm{d}$ y estuvo comprendido entre 28,8 y 165,2 g/d. Los valores de las pendientes en las ecuaciones obtenidas para los ácidos caproico, caprílico, cáprico, láurico, mirístico y palmítico (tabla III) indicaron que el efecto negativo de la grasa consumida sobre los ácidos grasos sintetizados ex novo en la ubre disminuye al acortarse la longitud de cadena, lo que coincide con resultados de investigaciones in vitro (Bauman y Davis, 1974). Estos resultados obtenidos también confirman observaciones previas de nuestro laboratorio (Martínez Marín et al., 2013).

El aumento lineal del contenido de ácido esteárico en la grasa láctea en respuesta al consumo de grasa (tabla III) puede explicarse porque cualquiera de los AGI de 18 átomos de carbonos de la dieta puede ser una fuente de dicho ácido por biohidrogenación ruminal (Palmquist et al., 2005). La respuesta del contenido de los AGI-C18 en la grasa láctea a las cantidades crecientes de grasa en la dieta fue mayor que la obtenida para el ácido esteárico. Este incremento podría deberse tanto a los AGI que escaparon intactos del rumen como, en el caso del oleico, a la $\Delta$-9 desaturación del ácido esteárico en la ubre (Chilliard y Ferlay, 2004; Bichi et al., 2012). Las pendientes de las ecuaciones de la tabla III indican que los incrementos de los contenidos del ácido esteárico y los AGI-C18 no compensaron la disminución de los de los ácidos grasos saturados de cadena media. Esto sugiere que los contenidos de otros ácidos grasos, probablemente cis- y trans-

Tabla III. Ecuaciones de regresión lineal (metarregresiones en la forma $Y=a+b$ * $X$ ) del efecto del consumo de grasa añadida a la dieta de ovejas (expresado en $\mathrm{g} / \mathrm{d}$ ) sobre los contenidos de ácidos grasos de la grasa láctea (Regression equations (meta-regressions in the form $Y=a+b{ }^{*} X$ ) of ewe's fat intake on fatty acid contents in milk fat).

\begin{tabular}{|c|c|c|c|c|c|c|c|c|c|}
\hline \multirow{2}{*}{$\begin{array}{l}\text { Ácidos grasos, } \\
\% \text { grasa láctea }\end{array}$} & \multicolumn{8}{|c|}{ Estimaciones de los parámetros } & \multirow[b]{2}{*}{$\mathrm{R}^{2}$} \\
\hline & $\mathrm{n}$ & a & EEM & $p$ & b & EEM & $\mathrm{p}$ & RECM & \\
\hline Caproico C6:0 & 59 & 2,880 & 0,1876 & $<0,001$ & $-0,0062$ & 0,00173 & 0,002 & 0,26 & 0,99 \\
\hline Caprílico C8:0 & 59 & 2,743 & 0,1746 & $<0,001$ & $-0,0084$ & 0,00179 & $<0,001$ & 0,34 & 0,98 \\
\hline Cáprico C10:0 & 61 & 8,450 & 0,4850 & $<0,001$ & $-0,0329$ & 0,00443 & $<0,001$ & 1,37 & 0,96 \\
\hline Láurico C12:0 & 61 & 4,889 & 0,2480 & $<0,001$ & $-0,0178$ & 0,00261 & $<0,001$ & 0,59 & 0,97 \\
\hline Mirístico C14:0 & 61 & 10,924 & 0,2857 & $<0,001$ & $-0,0252$ & 0,00317 & $<0,001$ & 1,06 & 0,95 \\
\hline Palmítico C16:0 & 61 & 26,01 & 0,6757 & $<0,001$ & $-0,0476$ & 0,00469 & $<0,001$ & 2,00 & 0,96 \\
\hline Esteárico C18:0 & 67 & 9,531 & 0,9023 & $<0,001$ & 0,0289 & 0,00643 & $<0,001$ & 2,56 & 0,94 \\
\hline AGI-C18 & 50 & 19,566 & 1,2658 & $<0,001$ & 0,0366 & 0,00510 & $<0,001$ & 2,15 & 0,98 \\
\hline IA & 50 & 4,069 & 0,2274 & $<0,001$ & $-0,0137$ & 0,00151 & $<0,001$ & 0,57 & 0,97 \\
\hline
\end{tabular}

$a=$ intersección; $b=$ pendiente; $E E M=$ error estándar de la media; $R E C M=$ raíz cuadrada del error cuadrático medio; $R^{2}=$ coeficiente de determinación. $\mathrm{C} 18 \mathrm{AGI}=$ suma de los ácidos oleico, linoleico y $\alpha$-linolénico. $I \mathrm{~A}=$ índice de aterogenicidad. (C12:0+4xC14:0+C16:0)/AGI-C18 (Ulbricht y Southgate,1991). 
mono y poliinsaturados procedentes del metabolismo ruminal de los ácidos grasos de la dieta, también aumentaron en respuesta al consumo de grasa suplementaria (Chilliard et al., 2007).

La reducción de los contenidos de los ácidos laúrico, mirístico y palmítico (AGSCM) y el aumento del contenido de los AGI-C18 ocasionó que el IA de la grasa láctea disminuyera linealmente con el consumo creciente de grasa suplementaria (tabla III). Este efecto es beneficioso desde el punto de vista de la salud humana porque las evidencias científicas indican claramente que el consumo de grasas ricas en AGSCM aumenta el riesgo de enfermedades cardiovasculares en las personas (Ulbricht y Southgate, 1991; Astrup et al., 2011).

\section{CONCLUSIONES}

La metarregresión permitió cuantificar la relación entre la grasa consumida y los cambios de los contenidos de los ácidos grasos estudiados en la grasa láctea de ovejas. La respuesta de los ácidos grasos de la leche de oveja al consumo creciente de grasa rica en ácidos grasos insaturados puede considerarse beneficiosos desde el punto el punto de vista de la salud humana, de acuerdo con los conocimientos científicos actuales.

\section{BIBLIOGRAFÍA}

Astrup, A.; Dyerberg, J.; Elwood, P.; Hermansen, K.; Hu, F.B.; Jakobsen, M.U.; Kok, F.J.; Krauss, R.M.; Lecerf, J.M.; LeGrand, P.; Nestel, P.; Risérus, U.; Sanders, T.; Sinclair, A.; Stender, S.; Tholstrup, T. and Willett, W.C. 2011. The role of reducing intakes of saturated fat in the prevention of cardiovascular disease: where does the evidence stand in 2010? Am J Clin Nutr, 93: 684-688.

Bauman, D.E. and Davis, C.L. 1974. Biosynthesis of milk fat. In: B.L. Larson and V.R. Smith (Eds.). Lactation: A comprehensive treatise. Volume 2. Academic Press. New York. pp. 31-75.

Bichi, E.; Toral, P.G.; Hervás, G.; Frutos, P.; Gómez-Cortés, P.; Juárez, M. and De la Fuente, M.A. 2012. Inhibition of 9desaturase activity with sterculic acid: effect on the endogenous synthesis of cis-9 18:1 and cis-9 trans-11 18:2 in dairy sheep. $J$ Dairy Sci, 95: 5242-5252.

Bouattour, M. 2007. Effects of feeding different vegetal fat sources to increase conjugated linoleic acid in milk of small ruminants and interaction with fibrolytic enzymes. Tesis Doctoral. Barcelona. España.

Caroprese, M.; Albenzio, M.; Bruno, A.; Fedele, V.; Santillo, A. and Sevi, A. 2011. Effect of solar radiation and flaxseed supplementation on milk production and fatty acid profile of lactating ewes under high ambient temperature. J Dairy Sci, 94: 3856-3867.

Castro, T.; Manso, T.; Jimeno, V.; Del Alamo, M. and Mantecón, A. R. 2009. Effects of dietary sources of vegetable fats on performance of dairy ewes and conjugated linoleic acid CLA in milk. Small Rum Res, 84: 47-53.

Chilliard, Y. and Ferlay, A. 2004. Dietary lipids and forages interactions on cow and goat milk fatty acid composition and sensory properties. Reprod Nutr Dev, 44: 467-492.

Chilliard, Y.; Glasser, F.; Ferlay, A.; Bernard, L.; Rouel, J. and Doreau,
M. 2007. Diet, rumen biohydrogenation and nutritional quality of cow and goat milk fat. Eur J Lipid Sci Technol, 109: 828855.

Chiofalo, B.; Liotta, L.; Zumbo, A. and Chiofalo, V. 2004. Administration of olive cake for ewe feeding: effect on milk yield and composition. Small Rum Res, 55: 169-176.

Cieslak, A.; Kowalczyk, J.; Czauderna, M.; Potkanski, A. and Szumacher-Strabel, M. 2010. Enhancing unsaturated fatty acids in ewe's milk by feeding rapeseed or linseed oil. Czech J Anim Sci, 55: 496-504.

De Oliveira Maia, M. 2011 . Efeito da adição de diferentes fontes de óleo vegetal na dieta de ovinos sobre o desempenho, a composição e o perfil de ácidos graxos na carne e no leite. Tesis Doctoral. Sao Paulo. Piracicaba. Brasil.

Gómez-Cortés, P.; Frutos, P.; Mantecón, A. R.; Juárez, M.; De la Fuente, M.A. and Hervás, G. 2008a. Milk production, conjugated linoleic acid content, and in vitro ruminal fermentation in response to high levels of soybean oil in dairy ewe diet. J. Dairy Sci, 91: 1560-1569.

Gómez-Cortés, P.; Frutos, P.; Mantecón, A. R.; Juárez, M.; De La Fuente, M.A. and Hervás, G. 2008b. Addition of olive oil to dairy ewe diets: Effect on milk fatty acid profile and animal performance. J Dairy Sci, 91: 3119-3127.

Gómez-Cortés, P.; Bach, A.; Luna, P.; Juárez, M. and De la Fuente, M.A. 2009. Effects of extruded linseed supplementation on $n-3$ fatty acids and conjugated linoleic acid in milk and cheese from ewes. J Dairy Sci, 92: 4122-4134.

Gómez-Cortés, P.; De la Fuente, M.A.; Toral, P.G.; Frutos, P.; Juárez, M. and Hervás, G. 2011 la. Effects of different forage: concentrate ratios in dairy ewe diets supplemented with sunflower oil on animal performance and milk fatty acid profile. J Dairy Sci, 94: 4578-4588

Gómez-Cortés, P.; Toral, P. G.; Frutos, P.; Juárez, M.; De La Fuente, M.A. and Hervás, G. 2011 b. Effect of the supplementation of dairy sheep diet with incremental amounts of sunflower oil on animal performance and milk fatty acid profile. Food Chem, 125: 644-651.

Hervás, G.; Luna, P.; Mantecón, Á.R.; Castañares, N.; de la Fuente, M.A.; Juárez, M. and Frutos, P. 2008. Effect of diet supplementation with sunflower oil on milk production, fatty acid profile and ruminal fermentation in lactating dairy ewes. $J$ Dairy Res, 75: 399-405.

Maia Ferreira, E. 2011. Óleo de peixe em substituição parcial ao óleo de soja em dietas para ovinos. Tesis Doctoral. Sao Paulo. Piracicaba. Brasil.

Martínez Marín, A.L.; Pérez Hernández, M.; Pérez Alba, L.M.; Carrión Pardo, D.; Gómez Castro, A.G.; Garzón Sigler, A.I. 2013. Effect of oils and seeds in ruminant diets on milk fat fatty acid profile. Review. Rev Mex Cienc Pecu, 4: 319-338.

Mele, M.; Buccioni, A.; Petacchi, F.; Serra, A.; Banni, S.; Antongiovanni, M. and Secchiari, P. 2006. Effect of forage/ concentrate ratio and soybean oil supplementation on milk yield, and composition from Sarda ewes. Anim Res, 55: 273-285.

Mughetti, L.; Sinesio, F.; Acuti, G.; Antonini, C.; Moneta, E.; Peparaio, M. and Trabalza-Marinucci, M. 2012. Integration of extruded linseed into dairy sheep diets: Effects on milk composition and quality and sensorial properties of Pecorino cheese. Anim Feed Sci Technol, 178: 27-39.

Paduan Nolli, C. 2012. Produção, composição do leite e desempenho reprodutivo de ovelhas Santa Inês alimentadas com rações contendo óleo de canola ou linhaça. Tesis Doctoral. Sao Paulo. Piracicaba. Brasil. 
Palmquist, D.L.; Lock, A.L.; Shingfield, K.J. and Bauman, D.E. 2005. Biosynthesis of conjugated linoleic acid in ruminants and humans. Adv Food Nutr Res, 50: 179-217.

Sales, J. and Homolka, P.A. 2011. Meta-analysis of the effects of supplemental dietary fat on protein and fibre digestibility in the horse. Livest Sci,136: 55-63.

Sauvant, D.; Schmidely, P.; Daudin, J.J. and St-Pierre, N.R. 2008. Meta-analyses of experimental data in animal nutrition. Animal, 2: 1203-1214.

Toral, P.G.; Frutos, P.; Hervás, G.; Gómez-Cortés, P.; Juárez, M. and De la Fuente, M.A. 2010a. Changes in milk fatty acid profile and animal performance in response to fish oil supplementation, alone or in combination with sunflower oil, in dairy ewes. $J$ Dairy Sci, 93: 1604-1615.

Toral, P.G.; Hervás, G.; Gómez-Cortés, P.; Frutos, P.; Juárez, M. and de La Fuente, M.A. $2010 \mathrm{~b}$. Milk fatty acid profile and dairy sheep performance in response to diet supplementation with sunflower oil plus incremental levels of marine algae. J Dairy Sci, 93: 1655-1667.

Ulbricht, T.L.V. and Southgate, D.A.T. 1991. Coronary heart disease: seven dietary factors. Lancet, 338: 985-992.

Zhang, R.; Mustafa, A.F. and Zhao, X. 2006a. Effects of flaxseed supplementation to lactating ewes on milk composition, cheese yield, and fatty acid composition of milk and cheese. Small Rum Res, 63: 233-241.

Zhang, R.H.; Mustafa, A.F. and Zhao, X. 2006b. Effects of feeding oilseeds rich in linoleic and linolenic fatty acids to lactating ewes on cheese yield and on fatty acid composition of milk and cheese. Anim Feed Sci Technol, 127: 220-233.

Zhang, R.H.; Mustafa, A.F. and Zhao, X. 2006c. Blood metabolites and fatty acid composition of milk and cheese from ewes fed oilseeds. Can J Anim Sci, 86: 547-556. 